\title{
BILATERAL BREAST HAMARTOMAS IN A YOUNG FEMALE - A CASE REPORT AND LITERATURE REVIEW OF THE UNDER RECOGNIZED BREAST LESION
}

\author{
Wijayapala MHWMN ${ }^{1}$, Perera LDRA ${ }^{2}$ \\ ${ }^{1}$ Department of Radiology, National Hospital of Sri Lanka, Sri Lanka \\ ${ }^{2}$ Department of Radiology, District General Hospital Kalutara, Sri Lanka
}

Keywords : Breast neoplasms; hamartoma; fibroadenolipoma

Corresponding Author: Wijayapala M HW $\mathrm{MN}^{1}$

Copyright: Wijayapala $M H W \mathrm{MN}^{1}$

iD https://orcid.org/0000-0002-5984-5118

\section{Introduction}

Breast hamartoma, also known as fibroadenolipoma is a rarely reported benign breast lesion of undefined pathogenesis ${ }^{1}$. The reported occurrence of hamartoma of the breast is $0.1-0.7 \%{ }^{2}$. They typically occur in females $>35$ years old ${ }^{1}$. Breast hamartomas are very rare in males ${ }^{3}$. Bilateral breast hamartomas are extremely infrequent. In the present report, however, a female with bilateral breast hamartomas is described with a literature review. Such a case has seldom been reported.

Due to poor awareness of breast hamartoma, the diagnosis is underestimated by clinicians, radiologists and pathologists. In addition to that, its diagnosis by a single radiological imaging modality is insufficient ${ }^{1}$. Misdiagnosis as fibroadenoma, even upon fine-needle aspiration (FNAC) is common ${ }^{1}$. Thus, the real prevalence may possibly be more than that reported ${ }^{1}$.

\section{Case Presentation}

A 34 years old woman who had painless lumps in both breasts, which had been progressively growing in size over 5 years duration was evaluated. She did not have any risk factors for breast cancer or significant familial factors. There was no prior breast trauma, surgery or radiation history. No skin/ nipple changes or pyrexia were noted. The patient had been investigated for the same complaint 2 years back, with sonography and FNAC, however both findings were in favor of fibroadenoma of the breasts.

On physical examination, oval, non-tender, smooth, mobile masses were identified in the upper outer quadrant of both breasts. No skin fixation, skin ulceration or nipple retractions were evident. 
Ultrasound of both breasts revealed well defined solid heterogeneous masses measuring $2.6 \times 2.3 \times 1.3 \mathrm{~cm}$ in $\mathrm{R} /$ breast 110 'clock position and $1.1 \times 1.1 \times 1.0 \mathrm{~cm}$ in $\mathrm{L} /$ breast 2 o'clock position. (Fig1 \& 2) Both lesions were parallel and there was no posterior acoustic shadowing. Vascularity is not detected in color Doppler USS.

Mammograms showed well-circumscribed oval mass lesions with thin capsules in both breasts upper outer quadrants. (Fig3 \& 4) They comprised of both fat and soft tissue internal densities producing breast within a breast appearance. No micro calcifications, stromal distortions or suspicious axillary nodes were detected.

With the intention of achieving diagnostic and therapeutic goals, complete surgical excisions of both breast hamartomas were performed. Histopathological diagnosis was found to be consistent with bilateral breast hamartomas. Microscopically, no cells with atypia were found. There was no recurrence in follow up imaging after 3 months.

\section{Discussion}

"Breast hamartoma is a benign proliferation of fibrous, glandular, and fatty tissue in normal or varying proportions enclosed by a thin connective tissue capsule" 4 .

The diagnosis is established by the results of mammography, ultrasonography and pathology investigations ${ }^{1}$. "Combination of these modalities is more accurate than the use of a single modality, which could result in misdiagnosis".

On mammography, breast hamartomas are depicted as well defined heterogeneous masses with radio opaque and radio transparent areas within it. This peculiar appearance is defined with various terms such as "bull's-eye, multilayered appearance, slice of sausage or breast within a breast", which is pathognomonic and virtually diagnostic ${ }^{3,2}$. Thin, radiopaque pseudo capsule and benign calcifications are other mammographic findings ${ }^{2}$.

Hamartomas composed predominantly fibrous tissue are difficult to differentiate from fibro adenomas $^{3}$. Hamartomas with high amounts of fatty tissue can simulate lipomas, lipo necrosis or oil cysts. Last two are almost always linked with histories of trauma or surgery, thus facilitating differential diagnosis ${ }^{3}$.

Since hamartomas resemble normal breast tissue, it is often challenging to delineate the margins ultrasonically ${ }^{4}$. It can be seen as a well-circumscribed, solid lesion with mixed internal echo texture, without micro calcification or posterior acoustic shadowing ${ }^{4}$. There is no hyper vascularity on color Doppler imaging ${ }^{3}$.

Typical breast hamartoma without suspicious features on ultrasonography or mammography is not an indication for $\mathrm{MRI}^{3}$. Heterogeneous signal intensity and a thin capsule are usually demonstrated on T1W and T2W sequences. Type I time/intensity curve showing gradual, progressive enhancement is depicted in post contrast images ${ }^{3}$. 
The limited records on the elastography describe that breast hamartomas are commonly less elastic than the adjacent breast tissues ${ }^{3}$. Yet, their consistency and compressibility are generally determined by the amount of fatty tissue they contain, which is greatly variable ${ }^{3}$.

Although considered benign, Breast hamartomas can transform in to in situ and infiltrating carcinomas very rarely ${ }^{5}$. Suspicious features include micro calcifications or spiculations on mammography, hypo echoic irregular masses on sonography ${ }^{3}$.

FNAC of hamartomas of the breast show significant overlap with other benign breast disease $^{6}$. It is often misdiagnosed as fibroadenoma on FNAC ${ }^{1}$. Hence, FNAC cannot confirm the diagnosis. Nevertheless, it is helpful to exclude malignancy ${ }^{7}$.

Despite growing slowly, it can grow bigger producing asymmetry of breast if timely excision is not done. Breast hamartomas can be cured by lumpectomy with only infrequent recurrence ${ }^{1}$.

"The presence of intact lobular units admixed with different quantities of fatty tissue and a comparative lack of stroma supports the diagnosis pathologically" ${ }^{8}$.This is beneficial to differentiate breast hamartoma from fibro adenoma ${ }^{8}$.

Since Cowden disease is associated with diffuse or bilateral mammary hamartomas, it is an important differential diagnosis in this case.

\section{Conclusions}

Breast hamartomas are benign. Hence when presented with characteristic radiological appearances, additional diagnostic procedures are not required. However, the diagnosis by a single Radiological imaging modality is difficult and inadequate. Recognition of suspicious imaging features that may be presenting within a breast hamartoma is important.

\section{Conflicts of interests}

Authors declare that there are no conflicts of interests.

Informed written consent was given by the patient for the publication of the case and images. 


\section{References}

1. Wang Z, He J. Giant breast hamartoma in a 41-year-old female: A case report and literature review. Oncol Lett. 2015;10(6):3719-3721. doi:10.3892/ol.2015.3794

2. Biren A S, Fareed R, Brandon B. Breast Hamartoma: Mammography and Magnetic Resonance Imaging. JOJ Case Stud. 2017; 4(5): 555646. DOI: 10.19080/JOJCS.2017.04.555646.

3. Presazzi A, Di Giulio G, Calliada F. Breast hamartoma: ultrasound, elastosonographic, and mammographic features. Mini pictorial essay. J Ultrasound. 2015;18(4):373-377. Published 2015 Jul 26. doi:10.1007/s40477-015-0175-0

4. https://radiopaedia.org/articles/breast-hamartoma

5. Scally N, Campbell W, Hall S, McCusker G, Stirling WJ. Invasive ductal carcinoma arising within a breast hamartoma. Ir J Med Sci. 2011;180:767-768. doi: 10.1007/s11845-009-04029

6. Mehrdad Herbert M.D. Michael Schvimer M.D. Sergei Zehavi M.D. Sonia Mendlovic M.D. Tami Karni M.D. Itzhak Pappo M.D. et al. Breast hamartoma: Fine-needle aspiration cytologicfinding.Cancercytopathology2003:99(4):255-258 https://doi.org/10.1002/cncr.11419

7. Breast Hamartoma: a Case Report December 2005Acta chirurgica Belgica 105(6):658-9 DOI: 10.1080/00015458.2005.11679798SourcePubMed Umut Barbaros,Ugur Deveci

8. Herbert M, Mendlovic S, Liokumovich P, Segal M, Zahavi S, Rath-Wolfson L, Sandbank J. Can hamartoma of the breast be distinguished from fibroadenoma using fine-needle aspiration cytology? Diagn Cytopathol. 2006;34:326-329. doi: 10.1002/dc.20404.

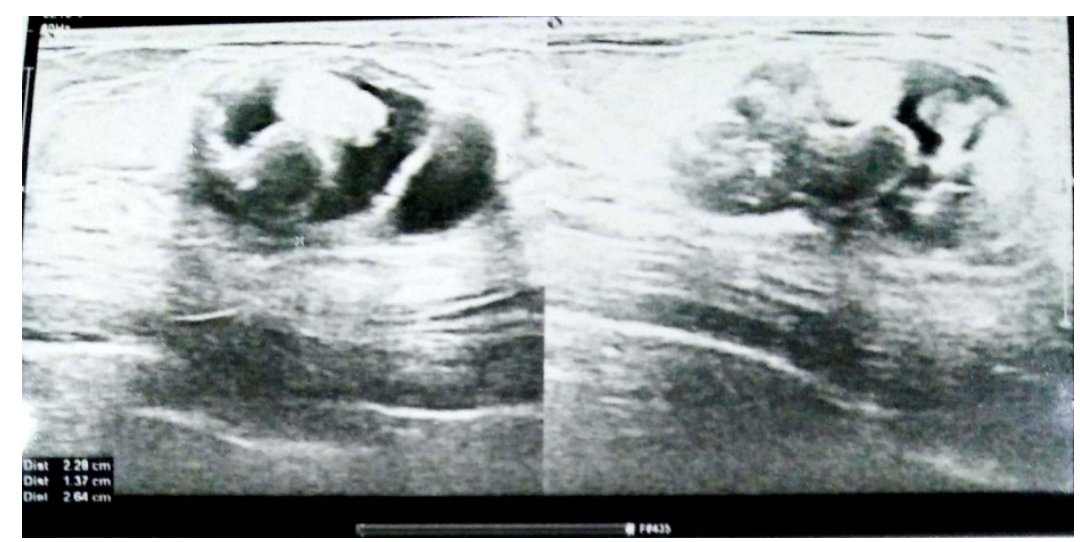

Figure 1: Ultrasonography of right breast upper outer quadrant showing a solid oval lesion with inhomogeneous echogenicity, which is typical of hamartoma. 


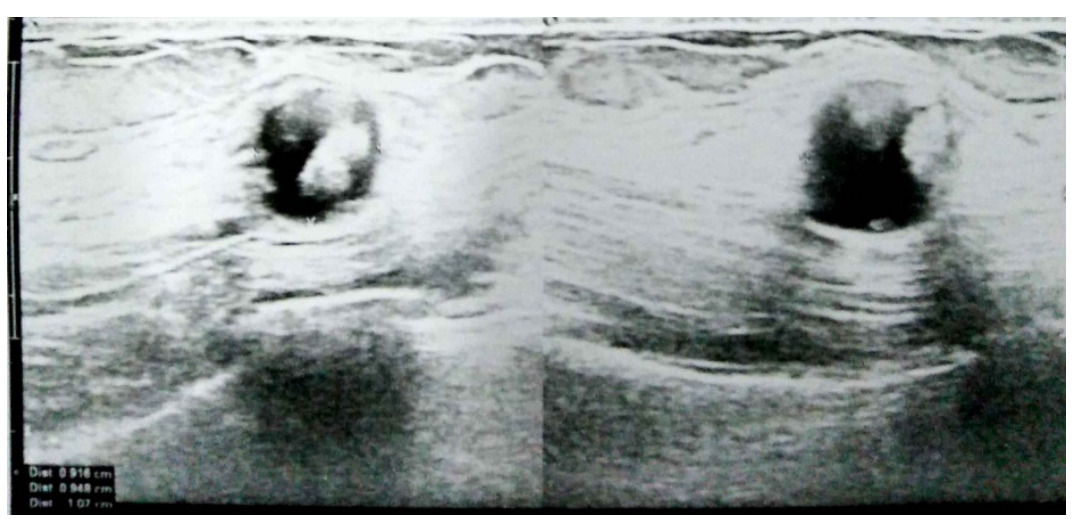

Figure 2: Ultrasonography of left breast upper outer quadrant showing a solid round lesion with mixed internal echo texture. This shows hypoechoic pseudo capsule clearly

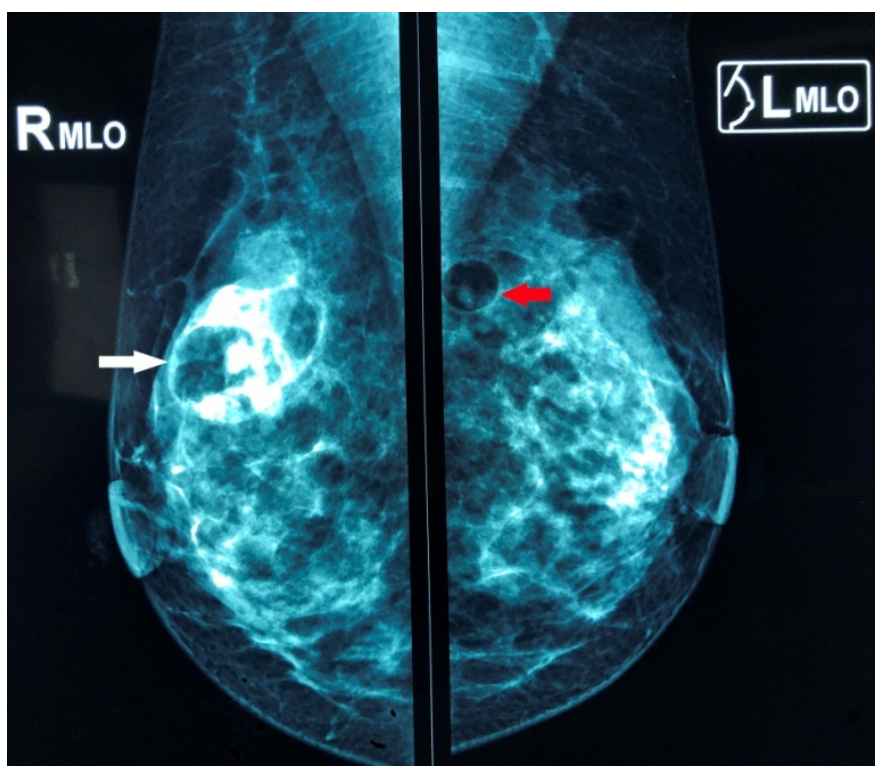

Figure 3 


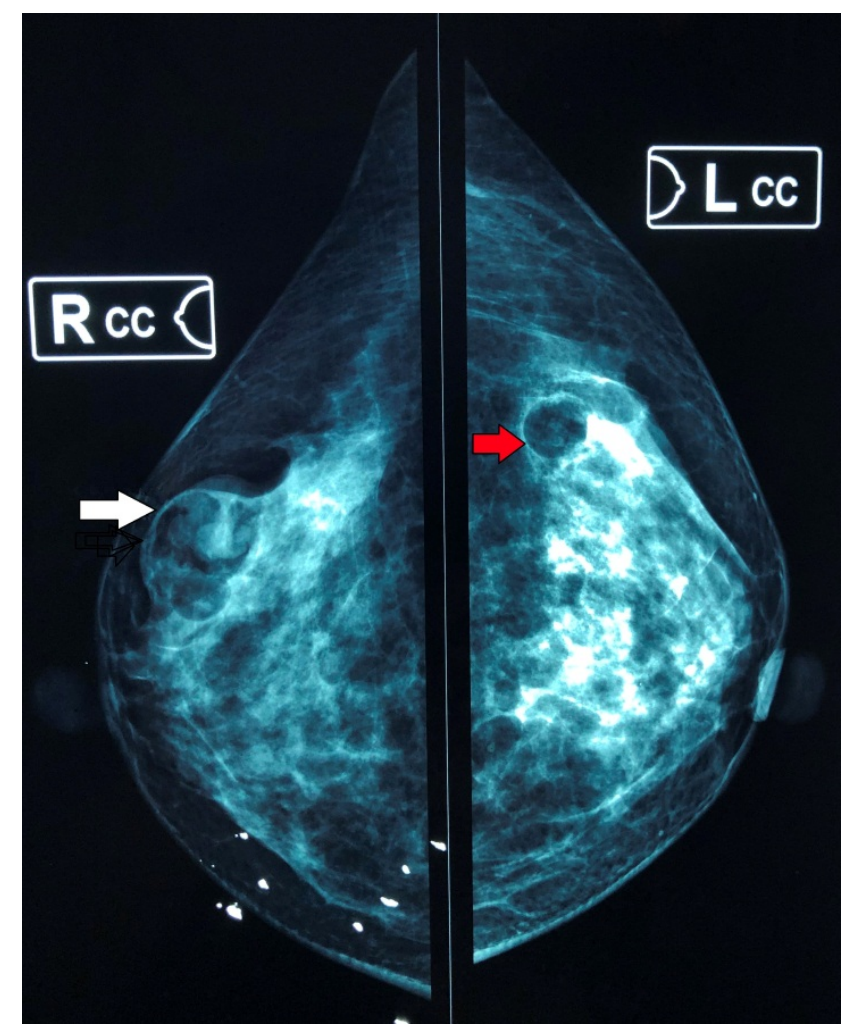

Figure 4

Figure 3 \& 4: Mammogram: MLO (Figure 3) and CC (Figure 4) views of B/L breasts show circumscribed masses containing fat and fibro glandular tissue at upper outer quadrants of both breasts. There is a radio-opaque thin line (pseudo capsule) surrounding the mass.

White arrow showing R/breast lesion, Red arrow showing L/breast lesion 